\title{
Monitoring of citrulline and diamine oxidase levels as biomarkers for intestinal mucositis during early-phase hematopoietic cell transplantation
}

\author{
Noriyasu Fukushima ${ }^{1,2,3}$, Satoshi Tomiyasu², Yoshinori Uji ${ }^{2}$, Masako Yokoo ${ }^{3}$, Takero Shindo ${ }^{3}$, Yasushi Kubota ${ }^{3}$, Toshihiko
} Ando $^{3}$, Kensuke Kojima ${ }^{3}$, Eisaburo Sueoka ${ }^{4}$, Tatsuo Ichinohe ${ }^{1}$, Shinya Kimura ${ }^{3}$

${ }^{1}$ Department of Hematology and Oncology, Research Institute for Radiation Biology and Medicine, Hiroshima University, Hiroshima, Japan. ${ }^{2}$ Department of Medical Science Technology, School of Health Sciences at Fukuoka, International University of Health and Welfare, Okawa, Japan ${ }^{3}$ Division of Hematology, Respiratory Medicine and Oncology, Department of Internal Medicine, Faculty of Medicine, Saga University, Saga, Japan. ${ }^{4}$ Department of Laboratory Medicine, Faculty of Medicine, Saga University, Saga, Japan.

\section{Abstract}

Background: Gastrointestinal tract mucositis is a common side effect in the early phase of hematopoietic cell transplantation (HCT), although reliable biomarkers have not yet been established. Since blood levels of citrulline and diamine oxidase (DAO) have been reported as specific markers of intestinal mucosal injury during cytotoxic cancer chemotherapy, we aimed to evaluate the relationship between circulating citrulline/DAO levels and the severity of gastrointestinal mucosal injury in patients with hematologic diseases who had undergone various types of HCT.

Methods: Forty patients who received autologous $(n=21)$ and allogeneic HCT $(n=19$; cord blood ( $n=16)$, peripheral blood $(n=3)$ ) were enrolled in the study. Serial monitoring of plasma citrulline and serum DAO levels was prospectively performed from the day prior to the start of the conditioning regimen until day 28 post-HCT.

Results: Citrulline and DAO levels significantly decreased after the start of conditioning. The recovery of citrulline and DAO levels tended to be delayed in cord blood transplant recipients, compared with autologous and allogeneic peripheral blood transplant groups, probably reflecting the severity of mucosal injury during the prolonged neutropenic period. The nadir levels for both markers were inversely associated with peak C-reactive protein levels.

Discussion: There was a slight difference in the time-dependent courses of these biomarker levels between each type of HCT. The change of DAO levels was more parallel to the clinical parameters. Serially monitored citrulline and DAO levels may be promising biomarkers for assessing intestinal mucosal injury in the early phase of various types of HCT, although prospective studies including larger number of patients are warranted to confirm our observations.

Key words: intestinal mucositis, hematopoietic stem cell transplantation, citrulline, diamine oxidase

Submitted December 19, 2017; Accepted July 7, 2018; Published online August 29, 2018; Issued online November 25, 2018

Correspondence: Noriyasu Fukushima, M. D., Ph.D, Department of Hematology and Oncology, Research Institute for Radiation Biology and Medicine, Hiroshima University, Kasumi 1-2-3, Minamiku,, Hiroshima, Hiroshima, 734-0037, Japan, E-mail: fukushin@ hiroshima-u.ac.jp

\section{Introduction}

Hematopoietic stem cell transplantation (HCT) is often associated with gastrointestinal complications. In patients who receive autologous or allogeneic HCT, digestive symptoms such as nausea, vomiting, diarrhea, and anorexia are frequent adverse events due to conditioning chemotherapy agents and/or total-body irradiation(TBI). In addition, graft-versus-host disease (GVHD), thrombotic microangiopathy (TMA), and infectious enteritis are major life-threatening problems for allogeneic HCT patients. In patients with hematological malignancies, these complications lead to impaired nutritional status during the course of $\mathrm{HCT}^{1-3}$, despite the absence of mal- 
Table 1. Patient characteristics

\begin{tabular}{|c|c|c|c|}
\hline & \multicolumn{2}{|c|}{ Allogeneic } & \multirow{2}{*}{$\begin{array}{c}\text { Autologous } \\
\text { PBSCT } \\
(n=21)\end{array}$} \\
\hline & $\begin{array}{c}\text { CBT } \\
(n=16)\end{array}$ & $\begin{array}{l}\text { PBSCT } \\
(n=3)\end{array}$ & \\
\hline Sex (male/female) & $12 / 4$ & $1 / 2$ & $10 / 11$ \\
\hline Age (average) & $22-64(55)$ & $28-66(44)$ & $22-66(51)$ \\
\hline \multicolumn{4}{|l|}{ Disease } \\
\hline AML & 4 & 2 & 1 \\
\hline ALL & 5 & & \\
\hline ATL & 2 & & \\
\hline $\mathrm{ML}$ & 4 & 1 & 13 \\
\hline Myeloma/amyloidosis & 1 & & 7 \\
\hline \multicolumn{4}{|l|}{ Conditioning } \\
\hline LEED & & & 5 \\
\hline MEAM & & & 7 \\
\hline HD-Mel & & & 6 \\
\hline Flu/Mel \pm TBI & 13 & 2 & \\
\hline $\mathrm{BU} / \mathrm{CY}$ or Flu $\mathrm{CY} \pm \mathrm{TBI}$ & 3 & 1 & \\
\hline Other & & & 2 \\
\hline \multicolumn{4}{|l|}{ GVHD prophylaxis } \\
\hline CsA & & 2 & \\
\hline Tacrolimus & 9 & 1 & \\
\hline MTX & 4 & 2 & \\
\hline MMF & 2 & & \\
\hline \multicolumn{4}{|l|}{ Disease status } \\
\hline $\mathrm{CR} / \mathrm{PR}$ & 4 & 1 & 7 \\
\hline not remission & 12 & 2 & 14 \\
\hline
\end{tabular}

Abbreviations: AML, acute myelogenous leukemia; ALL, acute lymphoblastic leukemia; ATL, adult T-cell leukemia/lymphoma; ML, malignant lymphoma; LEED, melphalan/etoposide/ cyclophosphamide/dexamethasone; MEAM, ranimustine/etoposide/cytarabine/melphalan; TBI, total body irradiation; HD-Mel, high dose-melphalan; Flu, fludarabine; BU, busulfan; CY, cyclophosphamide; PBSC, peripheral blood stem cell; GVHD, graft-versus-host disease; CsA, cyclosporine A; MTX, methotrexate; MMF, mycophenolate mofetil; CR, complete remission; $\mathrm{PR}$, partial remission

nutrition prior to preconditioning.

Citrulline, a free circulating amino acid, is preferentially synthesized by epithelial cells of the small intestine $^{4,5}$. The substrates for citrulline synthesis are amino acids obtained from the diet, such as glutamine. Most circulating citrulline is derived from glutamine conversion in enterocytes ${ }^{6}$. Plasma levels of citrulline serve as a biomarker of the functional small bowel enterocyte mass, independent of nutritional and inflammatory status ${ }^{4,5}$. Accordingly, plasma citrulline is a useful indicator of mucosal toxicity in myeloablative and non-myeloablative regimens followed by bone marrow and peripheral blood stem cell transplantation (PBSCT $)^{4,7,8}$. Recently, Hueso et al. suggested that citrulline levels in their study cohorts were a useful indicator to identify patients at risk of developing intestinal acute GVHD?

Diamine oxidase (DAO) is an endogenous enzyme that catabolizes a variety of substrates, including histamine and diamines ${ }^{10}$. Its main site of action is the small intestine, and DAO activity is especially high in the villi of the small intestinal mucosa ${ }^{11}$. The activity of DAO in the villi is an indicator of the severity of small intestinal mucosal injury due to autoimmune disease or drugs ${ }^{12-14}$. The relationship between chemotherapy-induced gastrointestinal tract toxicity and DAO activity has been previously investigated $^{13-16}$, although few studies have addressed its significance in the context of hematological malignancies ${ }^{16}$.

Here, we prospectively performed serial monitoring of blood citrulline and DAO levels and evaluated their relationship with the inflammatory response and the severity of gastrointestinal mucosal injury in patients with hematologic neoplasms undergoing various types of HCT during its early phase.

\section{Patients and methods}

\section{Patients}

A total of 40 patients who received autologous and allogeneic HCT in the department of Hematology, Respiratory Medicine and Oncology, Saga University Hospital, between April 2012 and March 2015 were enrolled in the study. Patient characteristics are summarized in Table 1. 
Of these patients, 21 received autologous PBSCT, while 19 received allogeneic HCT including 16 unrelated cord blood transplants (CBT) and 3 PBSCT from related donors. Two of these patients received allogeneic HCT because of a relapse after receiving autologous PBSCT. In autologous PBSCT, conditioning regimens were LEED (melphalan, cyclophosphamide, etoposide, and dexamethasone) or MEAM (ranimustine, etoposide, cytarabine, and melphalan) for patients with malignant lymphoma, and high-dose melphalan for patients with plasma cell myeloma or amyloidosis. Allogeneic HCT recipients received reduced-intensity conditioning regimens with or without TBI. The dose of TBI was $4 \mathrm{~Gy}$, except for one patient $(8 \mathrm{~Gy})$. Cyclosporine A or tacrolimus, with or without short-term methotrexate, was used for GVHD prophylaxis, depending on the underlying disease status and donor source. This study was performed in accordance with the Declaration of Helsinki, and the protocol was approved by the ethics committee of Saga University Hospital. All patients provided written informed consent.

\section{Measurement of plasma citrulline, serum DAO, and laboratory parameters}

Peripheral blood samples were collected prospectively from the day prior to the start of the conditioning regimen and on days 0 (the day of stem cell infusion), 7, 14, 21, and 28. Using these samples, plasma citrulline and serum DAO levels were measured in the following manner. Briefly, to evaluate plasma citrulline concentration, samples were ultracentrifuged to remove high-molecularweight proteins. Then, citrulline was separated on an Atlantis HILIC Column (Waters Corporation, Milford, MA, USA) and as mobile phase, using the ion-pairing agent, acetonitrile and a Waters Acuity UPLC H-class System (Waters Corporation). It was measured using tandem mass spectrometry on a Waters Acquity TQ detector. Hypocitrullinemia is defined as citrulline levels below $10 \mu \mathrm{mol} / \mathrm{L}$ and is considered to reflect severe GI mucositis ${ }^{17}$. Serum DAO levels were measured using an enzyme-linked immunosorbent assay (ELISA) kit (Immundiagnostik AG, Bensheim, Germany). Additionally, serum transferrin, transthyretin, retinol-binding protein (RBP), albumin, C-reactive protein ( $\mathrm{CRP}$ ), as well as absolute neutrophil and lymphocyte counts, were also measured each day. Transferrin, transthyretin, and RBP, which were termed rapid turnover proteins (RTPs) and are often used for nutritional assessment, were measured using turbidimetric immunoassay or latex agglutination photometric immunoassay. Other index data were obtained from routine laboratory examination.

\section{Assessment of clinical parameters}

Clinical parameters were assessed from the start of preconditioning until day 28. Basal energy expenditure (BEE), calculated using the Harris-Benedict equations, was assessed along with oral caloric intake, parenteral nutrition caloric intake, and degree of diarrhea. These data were obtained from patient questionnaires and medical records. In patients with a body temperature over $37.5^{\circ} \mathrm{C}$ and with diarrhea for several days, a differential diagnosis of the gastrointestinal symptoms was performed through screening for cytomegalovirus (CMV) antigenemia, Clostridium difficile toxin, and stool culture. When acute gastrointestinal GVHD was strongly suspected, intestinal mucosal biopsy was performed when possible. The degree of diarrhea was determined according to the Common Terminology Criteria for Adverse Events ver. 4.0.

\section{Statistical analysis}

We conducted a Friedman test for pairwise groups on each occasion, and then we conducted a Wilcoxon signed-rank post-hoc test to evaluate the differences. With respect to continuous variables, the Mann-Whitney U-test was used to compare the two groups. Data are presented as means \pm standard error. Pairwise correlations between parameters were assessed using Spearman's rank correlation test. A $p$-value $<0.05$ was considered significant. Analyses were performed with IBM SPSS Statistics software ver. 23 (SPSS Inc., Chicago, IL, USA).

\section{Results}

Change in citrulline levels during the early phase of various types of HCT

Citrulline levels significantly decreased over time following administration of preconditioning (pre) in all groups (Figure 1a). Mean baseline levels at preconditioning were $34.43 \pm 2.40 \mu \mathrm{mol} / \mathrm{L}$ and $31.55 \pm 3.05$ $\mu \mathrm{mol} / \mathrm{L}$, and nadir levels were $12.58 \pm 1.77 \mu \mathrm{mol} / \mathrm{L}$ (vs pre, $p<0.001$ ) on day 7 in the autologous PBSCT group and $13.37 \pm 1.77 \mu \mathrm{mol} / \mathrm{L}$ (vs pre, $p=0.001$ ) on day 14 in the CBT group (Figure 1a). Citrulline levels also declined in the allogeneic PBSCT group, but the difference relative to the other groups was not statistically significant due to the small number of patients. Recovery of citrulline levels tended to be delayed in the CBT group relative to autologous and allogeneic PBSCT groups, but there was no statistical significance. The number of patients with citrulline levels below $10 \mu \mathrm{mol} / \mathrm{L}$ on more than two occasions was 4 in the autologous PBSCT, 1 in the allogeneic PBSCT, and 5 in the CBT groups.

\section{Change in the DAO level during the early phase of various types of HCT}

Mean DAO levels decreased significantly from preconditioning to day $7(9.03 \pm 1.47$ to $5.79 \pm 0.65 \mathrm{U} / \mathrm{mL} ; p<$ 
(a)

$(\mu \mathrm{mol} / \mathrm{L})$

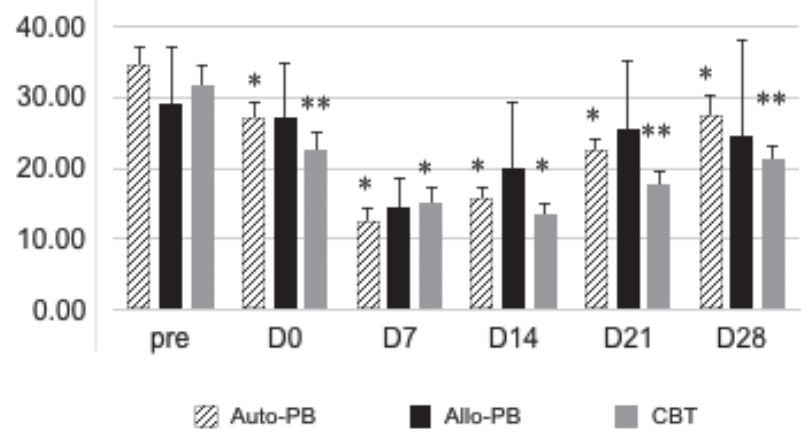

(b)

$(\mathrm{U} / \mathrm{mL})$

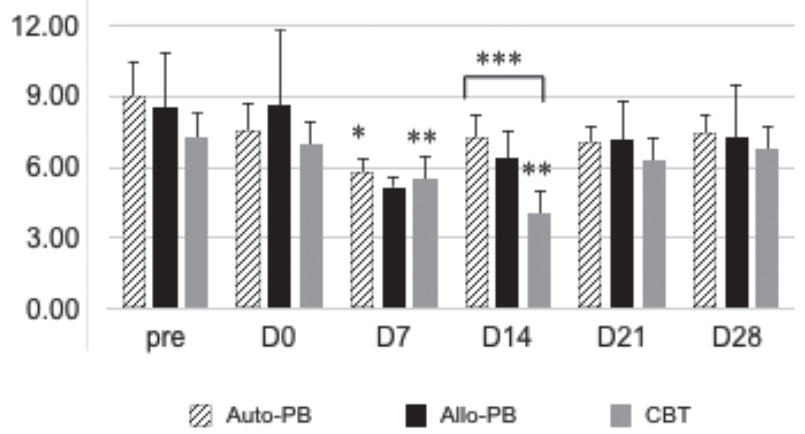

Figure 1. (a) The change in plasma citrulline levels after HCT. The level at each of the measuring points was significantly lower than before the day of pre-conditioning (pre) in the autologous PBSCT $(* p<0.001)$ and CBT groups $(* * p<0.01)$. The recovery of citrulline levels was delayed in the CBT group compared with the other groups. (b) Change in serum DAO levels after HCT. Nadir levels were significantly lower than before preconditioning autologous PBSCT ( $*$ at day $7: p<0.005$ ) and CBT groups (at day 7 and $14 * * p<0.01$ ). There is a significant difference between the levels in autologous PBSCT and CBT group at day $14 .(* * * p=0.01)$. Vertical bars present standard error.

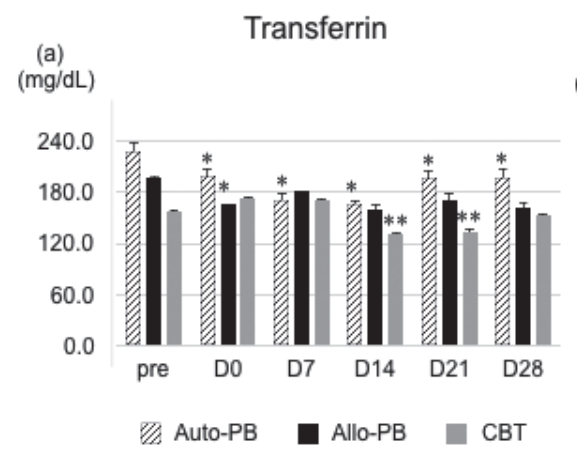

Albumin

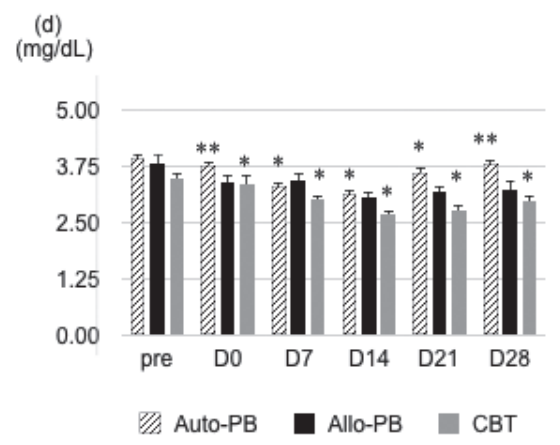

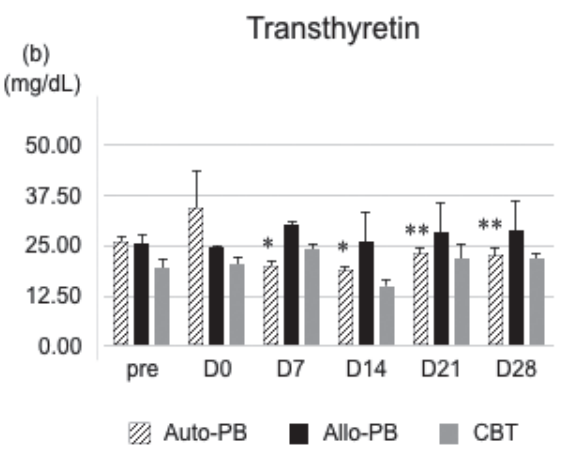

CRP

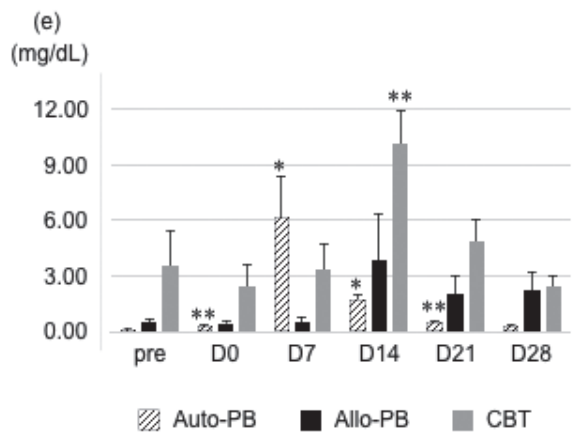

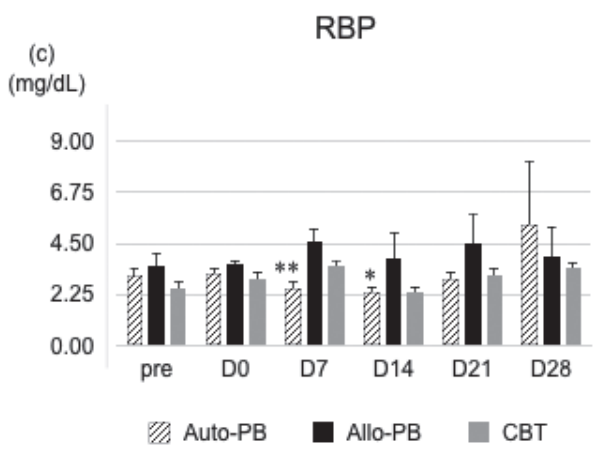

ANC

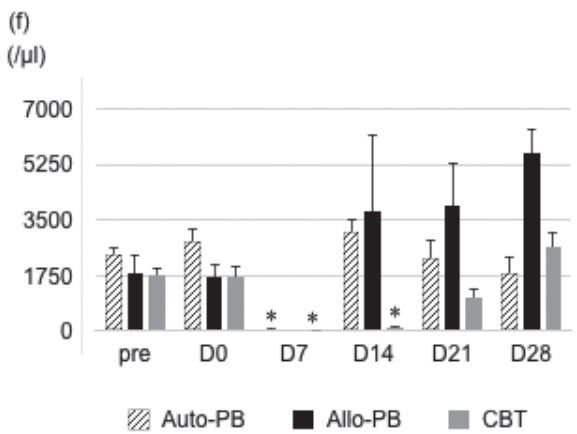

Figure 2. Changes in nutritional biomarkers, CRP, and neutrophil count during the course of the HCT: (a) transferrin (b) transthyretin (c) retino-binding protein (RBP) (d) albumin (e) CRP ( $\mathrm{f}$ ) neutrophil counts. ${ }^{*} p<0.01$ and ${ }^{*}{ }^{*} p<0.05$ indicate statistically significant difference between the day of pre-conditioning and the following time point. Vertical bars present standard error.

0.01 ), and then increased on day 14 in the autologous PBSCT group (Figure 1b). The time course of the DAO levels in the allogeneic PBSCT group was similar to that of the autologous PBSCT group. In contrast, the mean DAO level decreased significantly on day $14(4.02 \pm 0.57$ $\mathrm{U} / \mathrm{mL} ; p<0.001$ ) relative to the level at preconditioning $(7.31 \pm 1.05 \mathrm{U} / \mathrm{mL}$ ) (Figure 1b). Recovery of the DAO level was faster in the autologous and allogeneic PBSCT groups than in the CBT group, and the difference between groups on day 14 was statistically significant ( $p$ $=0.01)$. No significant correlations were observed between plasma citrulline concentrations and serum DAO levels at any time point (Tables S1 and S2). 


\section{Proportion of oral intake in total calories}

(\%)

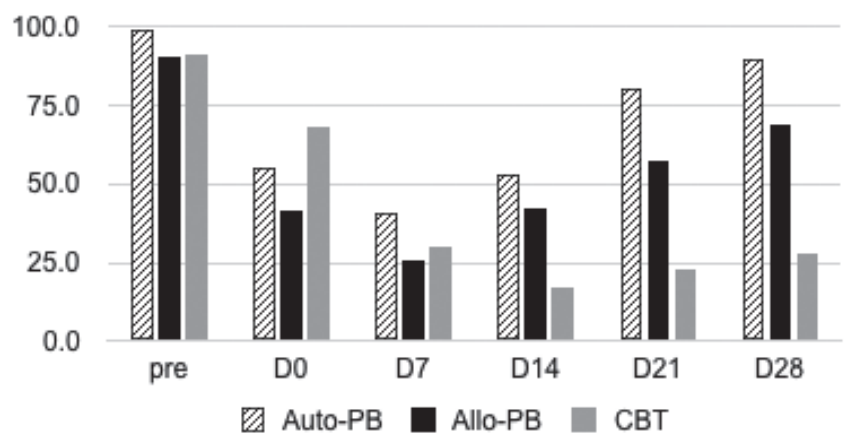

Frequency of patients with more than G2 diarrhea
(\%)

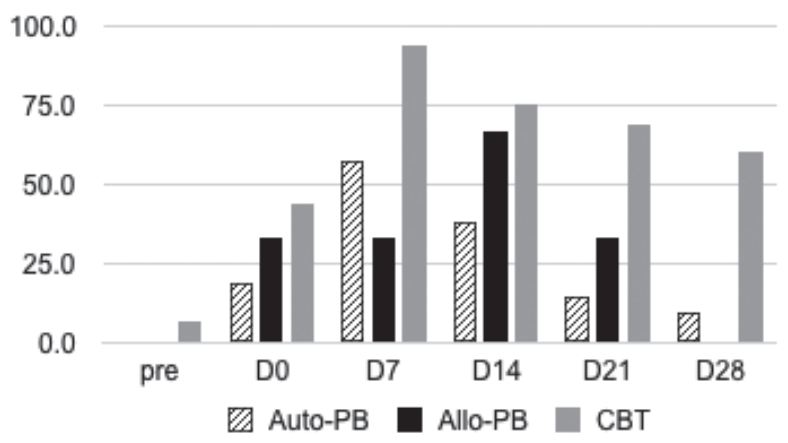

Figure 3. (a) Proportion of oral intake in total calories after HCT. Impairment of oral intake was observed at day 7 in all groups. Improvement of oral intake in the CBT group was delayed in comparison with the autologous and allogeneic PBSCT groups. (b) Frequency of patients with more than grade 2 diarrhea following HCT. More than half of the patients receiving CBT had diarrhea after day 21 , whereas day 21 most of the patients receiving autologous and allogeneic PBSCT had improved.

\section{Correlation between citrulline and DAO levels and laboratory parameters}

We observed the time course of nutritional assessment markers (RTPs and albumin) during the early phase of $\mathrm{HCT}($ Figure 2a-d). The levels of all markers in the CBT group were the lowest of all the groups and marker recovery was prolonged. There was no notable correlation between each mucosal injury biomarker and nutrition assessment, although there were correlations between citrulline levels and albumin on day 7 and 14 in the autologous PBSCT group (Tables S3-S6). The CRP peak occurred simultaneously with the nadir in citrulline and DAO levels (vs citrulline on day 7 in autologous group $(p$ $=0.003, p=-.621$; vs DAO on day 14 in the CBT group $(p=0.03, p=-.527)$, and on day 14 in the CBT group $(p<0.02, p=-.597)) \quad($ Figure 2e, Tables S3 and S4) . Neutropenia was prolonged in the CBT group relative to the autologous and the allogeneic PBSCT groups (Figure 2f). Specifically, the time course of citrulline levels was significantly correlated with the number of neutrophils in the CBT group (Table S3), but not in the other groups, suggesting that prolonged neutropenia might delay the recovery of intestinal integrity. DAO levels were not correlated with the time course of neutrophil count in any group (supplementary Table S3).

\section{Calorie intake profile and severity of diarrhea dur- ing HCT}

The ratio of total energy intake to BEE in each group was maintained at approximately $1: 1$ during HCT. The rate of oral intake, reflecting the severity of anorexia and oral mucositis, was significantly decreased in the CBT group in comparison with the autologous and allogeneic PBSCT groups (Figure 3a). In all groups, the change in the DAO levels was proportional to the rate of oral intake.
In contrast, citrulline levels had not recovered to pretransplant levels on day 21 in the autologous PBSCT group, despite the increase in the rate of oral intake. The rate of patients with more than grade 2 diarrhea was highest on day 7 in all groups (Figure 3b). Improvement of the severity grade was delayed in the CBT group relative to the autologous and allogeneic PBSCT groups. No onset of acute GVHD, TMA, CMV, or Clostridium difficilerelated enteritis was observed prior to day 28 . Half the patients receiving CBT still had diarrhea even after day 21, although their citrulline and DAO levels had increased from their nadir.

\section{Discussion}

Because resolution of intestinal mucosal injury is essential for the success of HCT, it is imperative to evaluate the severity of this morbid adverse event. Historically, small intestine endoscopy and biopsies have been considered the gold standard for the qualitative diagnosis of mucosal injury. However, it is sometimes difficult to perform endoscopic procedures due to their invasiveness and the fragility of recipients during the early phase of HCT. Many biomarkers have been considered for assessment of intestinal mucosal injury, but no standard approach for the use of such markers has yet been established. Previous studies have shown that sequential monitoring of DAO and citrulline levels is useful for evaluating intestinal mucosal injury in inflammatory bowel disease, ischemic intestinal disease, and cytotoxic chemotherapy ${ }^{18,19}$. Most of these studies showed that the degree of reduction in the levels of these substances reflects the severity of intestinal mucosal damage. However, we observed in this study that changes in the plasma levels of these molecules have unique characteristics in the early phase of 
HCT.

Citrulline levels also declined after the start of preconditioning, but there was no significant difference between the CBT and PBSCT groups over the time course, although the clinical symptoms and duration of neutropenia were more severe in the CBT group. LEED, MEAM, and high-dose melphalan, used in autologous PBSCT, are categorized as myeloablative regimens, whereas fludarabine plus melphalan (Flu-Mel) or BU + TBI 4 Gy, used in CBT, are both categorized as reduced-intensity regimens ${ }^{20}$. However, most CBT recipients in our study received TBI, as well as methotrexate to prevent GVHD, and both of these factors might have influenced the severity of mucosal toxicity ${ }^{21}$. Previous studies have reported that the changes in citrulline levels in PBSCT and bone marrow transplantation cohorts were dependent on the intensity of chemotherapy regimens ${ }^{22,23}$. Unfortunately, in our study, the number of patients receiving allogeneic PBSCT was small and we could not explain the significant difference between the other cohorts. Nonetheless, the change in citrulline levels in the allogeneic PBSCT group appeared to be similar to that of the autologous PBSCT group, when the intensity of conditioning regimens is stronger. The time course of the citrulline levels may be affected not only due to the intensity of preconditioning, but also due to the type of stem cell source or the duration of neutropenia. Further studies including larger number of patients are needed to clarify the factors associated with circulating citrulline levels.

In contrast, DAO activity followed a distinctive time course that clearly differed between PBSCT and CBT groups. The correlation between DAO levels and toxicities of chemotherapy has been extensively discussed in the field of gastrointestinal malignancy ${ }^{11-13}$, but, to the best of our knowledge, it has never been examined in the context of treatment of hematologic neoplasms including $\mathrm{HCT}^{16}$. DAO levels also decreased after the start of conditioning but recovered significantly faster in the PBSCT group than in the CBT group, unlike citrulline. In addition, the change in DAO levels was more synchronized with clinical symptoms, including the duration of diarrhea and the rate of oral intake during the acute phase of HCT. Therefore, DAO levels may be related to the severity of clinical symptoms.

Although DAO and citrulline levels are recognized as surrogate markers of a total enterocyte mass, the behavior of these markers appears to be different after intestinal mucosal injury occurs. Previous studies have shown that plasma DAO levels increase at the onset of mesenteric ischemia. Cakmaz et al. measured plasma DAO levels and citrulline in Wistar albino rats with acute mesenteric ischemia $^{19}$. They found that DAO levels significantly increased in a time-dependent manner, whereas citrulline levels significantly decreased after intestinal ischemia.
Citrulline levels have also been reported to decrease in any intestinal mucosal injury ${ }^{4,5}$. However, DAO concentration varies depending on physiological factors and the length of the regimen, in accordance with the intensity of preconditioning ${ }^{4,5,19}$. Although the mechanisms by which the mucosal injuries develop might differ between artificial mesenteric ischemia and chemotherapy-induced intestinal toxicity, these biomarkers may reflect different aspects of the cellular regeneration process. Further investigation is required to elucidate unparalleled kinetics in the time course of these biomarkers.

Finally, because intestinal mucositis is associated with malnutrition and acute phase inflammation during HCT, we speculated that levels of nutritional markers and acute inflammatory proteins would be strongly associated with DAO and citrulline levels ${ }^{24}$. However, we could not detect any significant correlation between nutrition assessment markers and mucosal injury markers, although we found that the nadir levels of these markers were inversely associated with peak CRP levels. The relationship between acute inflammatory markers and mucosal injury markers remains controversial. Some studies have reported that citrulline levels are inversely correlated with $\mathrm{CRP}^{22,25,26}$, whereas Gosselin reported the opposite result ${ }^{27}$. CRP is not a specific tissue injury marker often influenced by other factors, but it still should be considered as a potential robust biomarker because it is easily and reliably measured across diagnostic laboratories. In contrast, DAO and citrulline levels are likely to be more specific for intestinal mucosal damage. Simultaneously, most patients had diarrhea, and neutropenia showed an inverse correlation at the time of nadir levels of these two markers especially in the CBT group. Collectively, our results suggest that it is feasible to evaluate the severity of gastrointestinal mucosal damage in a more detailed manner through monitoring DAO and citrulline levels post-HCT using different stem cell sources.

\section{Conclusion}

Blood citrulline and DAO levels can be useful biomarkers for assessing intestinal mucosal injury during the early phase of HCT. The time course of citrulline levels may reflect the intensity of the preconditioning regimen, whereas DAO levels may be more related to clinical symptoms. Furthermore, when assessing intestinal mucosal injury, it is necessary to consider the type of stem cell source as well as the conditioning regimens that patients have received, using these biomarkers after autologous and allogeneic HCT.

\section{Acknowledgment}

We thank Reiko Ando, Ayako Aihara, Chiori Kantake, 
Ritsuko Iwakiri, Aya Madea, and the nursing team for data management and preparation of material. This study was supported by Grants-in-Aid for Scientific Research from the Ministry of Education, Culture, Sports, Science, and Technology of Japan. (25461454 to NF), Grants-inAid from the Japan Agency for Medical Research and Development (AMED) (\#17ek05100022h0001 to TI), and the program of the network-type Joint Usage/ Research Center for Radiation Disaster Medical Science of Hiroshima University, Nagasaki University, and Fukushima Medical University.

\section{Author's contributions}

Conception and design: N. Fukushima

Measurement of samples: N. Fukushima, M. Yokoo, S. Tomiyasu, and Y. Uji

Acquired and managed patients: N. Fukushima, T. Shindo, Y. Kubota, and T. Ando

Analysis and interpretation of data: N. Fukushima and T. Ichinohe

Writing, review and/or revision of the manuscript: N. Fukushima and T. Ichinohe

Study supervision: K. Kojima, E. Sueoka, and S Kimura

\section{Conflict of interest}

No authors have relevant conflicts of interest to declare. Disclosure forms provided by the authors are available here.

\section{References}

1. Toro JJ, Haile DJ, Chao JH, Schneider D, Jewell PS, Lee S, et al. The department of veterans' affairs nutritional status classification scheme allows for rapid assessment of nutritional status prior to the autologous peripheral blood stem cell transplantation and identifies patients at high risk of transplantrelated complications. Biol Blood Marrow Transplant. 2009; 15: $1060-5$.

2. Hagiwara S, Mori T, Tuchiya H, Sato S, Higa M, Watahiki M, et al. Multidisciplinary nutritional support for the autologous hematopoietic stem cell transplantation: A cost-benefit analysis. Nutrition. 2011; 27: 1112-7

3. Imataki O, Nakatani S, Hasegawa T, Kondo M, Ichihashi K, Araki M, et al. Nutritional support for patients suffering from intestinal graft-versus-host disease after the allogeneic hematopoietic stem cell transplantation. Am J Hematol. 2006; 81: 74752.

4. Barza JA, Szczylik C, Rzepecki P, Jaworska M, Anuszewska E. Plasma citrulline level as a biomarker for cancer therapyinduced small bowel mucosal damage. Acta Biochim Pol.

\section{4; 61: 615-31}

5. Banerjee A. Gastrointestinal toxicity biomarkers In: Gupta RC eds. Biomarkers in Toxicology, 1st edition, Amsterdam, Netherlands, Academic Press, Elsevier. 2014, 269-77.

6. Wu G. Intestinal mucosal amino acid catabolism. J Nutr. 1998; 128: $1249-52$.

7. Blijlevens NM, Lutgens LC, Schattenberg AV, Donnelly JP. Citrulline: a potentially simple quantitative marker of intestinal epithelial damage following myeloablative therapy. Bone Marrow Transplant. 2004; 34: 193-6.

8. Jordan K, Pontoppidan P, Uhlving HH, Kielsen K, Burrin DG, Weischendorff $\mathrm{S}$, et al. Gastrointestinal toxicity, systemic inflammation, and liver biochemistry in allogeneic hematopoietic stem cell transplantation. Biol Blood Marrow Transplant. 2017; 23: 1170-76.

9. Hueso T, Coiteux V, Joncquel Chevalier Curt M, Labreuche J, Jouault T, Yakoub-Agha I, et al. Citrulline and monocytederived macrophage reactivity before conditioning predict acute graft-versus-host disease. Biol Blood Marrow Transplant. 2017; 23: 913-21.

10. McGrath AP, Hilmer KM, Collyer CA, Shepard EM, Elmore BO, Brown DE, et al. Structure and inhibition of human diamine oxidase. Biochemistry. 2009; 48: 9810-22.

11. Luk GD, Bayless TM, Baylin SB. Plasma postheparin diamine oxidase. Sensitive provocative test for quantitating length of acute intestinal mucosal injury in the rat. J Clin Invest. 1983; 71: 1308-15.

12. Honzawa Y, Nakase H, Matsuura M, Chiba T. Clinical significance of serum diamine oxidase activity in inflammatory bowel disease: Importance of evaluation of small intestinal permeability. Inflamm Bowel Dis. 2011; 17: E23-5.

13. Moriyama K, Kouchi Y, Morinaga H, Irimura K, Hayashi T, Ohuchida A, et al. Diamine oxidase, a plasma biomarker in rats to GI tract toxicity of oral fluorouracil anti-cancer drugs. Toxicology. 2006; 217: 233-9.

14. Miyoshi J, Miyamoto H, Goji T, Taniguchi T, Tomonari T, Sogabe M, et al. Serum diamine oxidase activity as a predictor of gastrointestinal toxicity and malnutrition due to anticancer drugs. J Gastroenterol Hepatol. 2015; 30: 1582-90

15. Namikawa T, Fukudome I, Kitagawa H, Okabayashi T, Kobayashi M, Hanazaki K. Plasma diamine oxidase activity is a useful biomarker for evaluating gastrointestinal tract toxicities during chemotherapy with oral fluorouracil anti-cancer drugs in patients with gastric cancer. Oncology. 2012; 82: 14752.

16. Tsujikawa T, Uda K, Ihara T, Inoue T, Andoh A, Fujiyama Y, et al. Changes in serum diamine oxidase activity during chemotherapy in patients with hematological malignancies. Cancer Lett. 1999; 147: 195-8.

17. Crenn P, Vahedi K, Lavergne-Slove A, Cynober L, Matuchansky C, Messing B. Plasma citrulline: A marker of enterocyte mass in villous atrophy-associated small bowel disease. Gastroenterology. 2003; 124: 1210-9.

18. Kong W, Wang J, Ping X, Shen J, Ni X, Liu F, et al. Biomarkers 
for assessing mucosal barrier dysfunction induced by chemotherapy: Identifying a rapid and simple biomarker. Clin Lab. 2015; 61: 371-8.

19. Cakmaz R, Büyükaşık O, Kahramansoy N, Erkol H, Cöl C, Boran $\mathrm{C}$, et al. A combination of plasma DAO and citrulline levels as a potential marker for acute mesenteric ischemia. Libyan J Med. 2013; 8: 1-6.

20. Bacigalupo A, Ballen K, Rizzo D, Giralt S, Lazarus H, Ho V, et al. Defining the intensity of conditioning regimens: working definitions. Biol Blood Marrow Transplant. 2009; 15: 1628-33

21. Chaudhry HM, Bruce AJ, Wolf RC, Litzow MR, Hogan WJ, Patnaik MS, et al. The Incidence and Severity of Oral Mucositis among Allogeneic Hematopoietic Stem Cell Transplantation Patients: A Systematic Review. Biol Blood Marrow Transplant. 2016; 22: 605-16.

22. van der Velden WJ, Herbers AH, Brüggemann RJ, Feuth T, Donnelly JP, Blijlevens NM. Citrulline and albumin as biomarkers for gastrointestinal mucositis in recipients of hematopoietic SCT. Bone Marrow Transplant. 2013; 48: 977-81.

23. Herbers AH, Feuth T, Donnelly JP, Blijlevens NM. Citrullinebased assessment score: first choice for measuring and moni- toring intestinal failure after high-dose chemotherapy. Ann Oncol. 2010; 21: 1706-11.

24. Rzepecki P, Barzal J, Oborska S. Blood and marrow transplantation and nutritional support. Support Care Cancer. 2010; 18 Suppl 2: S57-65.

25. Blijlevens NM, Donnelly JP, DePauw BE. Inflammatory response to mucosal barrier injury after myeloablative therapy in allogeneic stem cell transplant recipients. Bone Marrow Transplant. 2005; 36: 703-7.

26. Pontoppidan PL, Jordan K, Carlsen AL, Uhlving HH, Kielsen $\mathrm{K}$, Christensen $\mathrm{M}$, et al. Associations between gastrointestinal toxicity, micro RNA and cytokine production in patients undergoing myeloablative the allogeneic stem cell transplantation. Int Immunopharmacol. 2015; 25: 180-8.

27. Gosselin KB, Feldman HA, Sonis AL, Bechard LJ, Kellogg $\mathrm{MD}$, Gura K, et al. Serum citrulline as a biomarker of gastrointestinal function during hematopoietic cell transplantation in children. J Pediatr Gastroenterol Nutr. 2014; 58: 709-14.

https://doi.org/10.31547/bct-2017-002

Copyright @ 2018 APBMT. All Rights Reserved. 